\title{
Total Variation for the Analysis of Event-Related Potentials
}

\author{
Alexander Klein*, Wolfgang Skrandies
}

4th April 2016

Physiologisches Institut der Justus-Liebig-Universität Gießen

Aulweg 129, D-35392 Gießen

\begin{abstract}
Event-related potential waveforms are often analysed in the timedomain for changes of striking morphological features, like amplitudes or latencies of extrema, at the expense of missing less obvious changes in overall morphology. In this article, we explore the applicability of the analysis of total variation to such waveforms, which makes it possible to capture overall changes in their morphology. We explain the concept of total variation from a theoretical point of view, followed by the application of the method to data from two learning experiments, which had previously proved difficult to analyse by more conventional methods. The results suggest that the analysis of total variation can be an interesting complement to more traditional analyses.
\end{abstract}

Keywords Total variation, event-related potential, time-domain, learning, feedback

\section{Introduction}

Event-related potential waveforms (ERPs) are commonly analysed in the timedomain for prima facie features of the curve, like peak amplitudes and latencies, or their respective differences. In the frequency-domain, on the other hand, it is possible to analyse ERPs as a whole for changes in amplitude or phase at certain frequencies.

Conventional methods in the time-domain are usually completely ignorant with respect to features in the frequency-domain and vice versa. It is usually not possible, for example, to detect changes of frequency content by analysing amplitudes, latencies, or simple moment estimates like mean and variance,

*Corresponding author: alexander.klein@physiologie.med.uni-giessen.de 
whereas in a Fourier-analysis, shifts in peak-latency could only be detected as obscure changes of phase over the whole range of frequencies, which may remain undetected, as the phase-spectrum is often not looked at, leaving only changes of the amplitude-spectrum as a subject of analysis.

Wavelet- and Gabor-analysis or similar methods [Witte et al., 2008, Wacker and Witte, 2013] may provide a way out of this dilemma by merging time- and frequency-domain analysis, but doing statistics correctly may prove challenging in this context [Maraun et al., 2007], and it is not easy to decide what exactly to search for: Complex wavelets provide amplitude- and phase-scalograms, but while the information provided by the former is quite obvious, as in their simplest form they can indicate, whether a certain "frequency" is "present" at a given time, the latter are only poorly understood, although useful information can be inferred from them [Deng et al., 2005a,b].

While all these are well-established methods of analysis, many of them tend to focus very narrowly on specific features like peaks and their latencies, perhaps within given regions of interest, while ignoring everything else. On the other hand, trying to analyse ERPs as a whole by simply treating them as stochastic processes and estimating their moments is not effective, either, as moment estimates have difficulty representing the overall morphology of a signal.

ERPs often show considerable interindividual variation. Aside from obvious differences in mean, variance, extremal amplitudes, and feature latencies, there may also be differences in the general shape of components: temporal dilatation or contraction may occur, additional superimposed fast undulations might undergo changes in amplitude, frequency or phase, or additional local extrema on the shoulders of larger components might occur or change. Such changes may occur alone or simultaneous, and may or may not influence the above measures.

After all, it seems desirable to have an additional measure quantifying overall signal morphology, as opposed to measures for specific features only. We think that one useful descriptor for such a task is a measure called "total variation". In the following, we will give an analytical definition of this measure, work out a few analytical examples, show a few simulations comparing its performance to other measures, and finally apply it for the analysis of data taken from two EEG experiments. The results show that the analysis of total variation may provide additional insight into morphological changes of signals that might otherwise remain unnoticed, thus complementing traditional methods of analysis with little additional effort in computation.

Although the concept of total variation is by no means new, it seems to have largely gone unnoticed as a general means for signal analysis. The concept itself appears under the name "short time line length" in Dümpelmann et al. [2012, p. 1723] as a means for detection of high frequency ripples in epilepsy, and a similar concept has been used as an intermediate step for fractal analyses of waveforms [Katz, 1988]. However, we currently know of no application of the measure itself for the evaluation and comparison of signal morphologies. 


\section{Analytical Total Variation}

\subsection{Definition and elementary properties}

Let $[a, b] \subset \mathbb{R}$ be an interval and $f:[a, b] \rightarrow \mathbb{R}$ any function, then the total variation of $f$ is defined as [Sohrab, 2003, p. 284, Def. 7.6.1]

$$
\sup \left\{\sum_{k=1}^{n}\left|f\left(x_{k}\right)-f\left(x_{k-1}\right)\right|: n \in \mathbb{N}, a=x_{0}<\ldots<x_{n}=b\right\} .
$$

For any continuously differentiable $f$ on an interval $[a, b] \subset \mathbb{R}$, the total variation can be calculated as [Sohrab, 2003, p. 295]

$$
V_{[a, b]}(f):=\int_{a}^{b}\left|f^{\prime}(x)\right| d x .
$$

These somewhat abstract formulæ can be explained immediately, when we restrict ourselves to continuously differentiable functions with only a handful of extrema ("smooth functions") for the sake of simplicity:

Suppose we have a function $f:[a, b] \mapsto \mathbb{R}$ with $n$ extrema at $x_{1}, \ldots, x_{n} \in$ $(a, b) \in \mathbb{R}$ - which are the points where $f^{\prime}$ changes its sign, and thus maxima or minima occur - and set additionally $x_{0}=a, x_{n+1}=b$. In this case we can write

$$
\int_{a}^{b}\left|f^{\prime}(x)\right| d x=\sum_{k=1}^{n+1}\left|f\left(x_{k}\right)-f\left(x_{k-1}\right)\right|,
$$

which is just the sum of the differences of the ordinates at adjacent extremal points including the borders, or in other words, the total distance an imaginary pen drawing the curve would travel in $y$-direction. As an immediate consequence, we see that a second function $g:[\bar{a}, \bar{b}] \mapsto \mathbb{R}$ with $n$ extrema at $\bar{x}_{1}, \ldots, \bar{x}_{n} \in(\bar{a}, \bar{b})$ and $f\left(x_{k}\right)=g\left(\bar{x}_{k}\right)$ for all $k$ will have the same total variation, although the intervals $[a, b]$ and $[\bar{a}, \bar{b}]$ may differ in length.

In the case of digitised data $D=\left(d_{0}, \ldots, d_{n}\right)$, we do not need to determine any extrema at all, as we can approximate Formula (1) directly: Setting $\Delta$ as the operator computing the first order differences $d_{k}-d_{k-1}$, and remembering that the 1 -Norm is defined as $\left\|\left(y_{1}, \ldots, y_{n}\right)\right\|_{1}:=\sum_{k=1}^{n}\left|y_{k}\right|$, we get the following approximation of Formula $(1)^{1}$ :

$$
V_{[a, b]} f \approx\|\Delta D\|_{1},
$$

because $\Delta$ approximates the first derivative, and $\|\cdot\|_{1}$ approximates the integral, both with reciprocal factors of proportionality. We can therefore estimate $V_{[a, b]} f$ directly from the recorded data by means of the simple command norm ( $\operatorname{diff}(D), 1$ ) in GNU-Octave [Octave community, 2012], for example.

\footnotetext{
${ }^{1}$ Note, however, that the quality of approximation is dependent on the smoothness of the data, which is directly related to the sampling frequency: The formulæ work well when the maximal frequency in the data is well below the Nyquist limit, as is the case for the data analysed in this paper and probably most real recordings of EEG or ERP signals, but results will get worse as high frequencies and noise creep up, in which case more elaborate approximations would have to be used.
} 


\section{$2.2 \quad$ Examples}

\subsubsection{Analytical examples}

A polynomial Let $f(x):=(x-1) \cdot(x-2) \cdot(x-3)=x^{3}-6 x^{2}+11 x-6$. What is the total variation of $f$ on $[0,4]$ ?

After differentiating we get $f^{\prime}(x)=3 x^{2}-12 x+11$, and the extremal abscissæ can be determined as the zeroes thereof, which are $x_{1,2}=2 \pm 1 / \sqrt{3}$. Using Formula (2) we get

$$
\begin{aligned}
V_{[0,4]} f & =\sum_{k=1}^{3}\left|f\left(x_{k}\right)-f\left(x_{k-1}\right)\right| \\
& =\left|\frac{2}{3 \sqrt{3}}-(-6)\right|+\left|-\frac{2}{3 \sqrt{3}}-\frac{2}{3 \sqrt{3}}\right|+\left|6-\left(-\frac{2}{3 \sqrt{3}}\right)\right| \\
& =12+\frac{8}{3 \sqrt{3}} \approx 13.54 .
\end{aligned}
$$

Harmonic oscillations Let $g(x):=\sin (k \cdot x), k \in \mathbb{N}$. What is the total variation of $g$ on an interval $[a, b] \subset \mathbb{R}$ ?

We simplify the calculations somewhat by first noting that the total variation of a complete cycle of a (co)sine is 4 , and that a complete cycle of $g$ happens on an interval of length $2 \pi / k$. Now let $x_{a}:=\lceil a / 2 \pi / k\rceil \cdot 2 \pi / k \geq a$ and $x_{b}:=\lfloor b / 2 \pi / k\rfloor \cdot 2 \pi / k \leq$ $b$ the first start and last end, respectively, of such a complete cycle in the vicinity of $[a, b]$, and $n:=\left(x_{b}-x_{a}\right) / 2 \pi / k \in \mathbb{Z}, n \geq-1$. Basically, $n$ counts the number of complete cycles in $\left[x_{a}, x_{b}\right]$, but since we never required $x_{a}<x_{b}, n$ may take the values -1 or 0 as well. Putting the pieces together, we get

$$
V_{[a, b]} g=\int_{a}^{x_{a}} k|\cos k x| d x+4 n+\int_{x_{b}}^{b} k|\cos k x| d x .
$$

In order to work out an example, take $a:=0.5, b:=17.4$ and $k:=7$. In this case we have $x_{a}=1 \cdot 2 \pi / 7, x_{b}=19 \cdot 2 \pi / 7$ and $n=18$. The final result is thus

$$
\begin{aligned}
V_{[0.5,17.4]} \sin 7 x & =\int_{0.5}^{2 \pi / 7} 7|\cos 7 x| d x+4 \cdot 18+\int_{19.2 \pi / 7}^{17.4} 7|\cos 7 x| d x \\
& \approx 1.65+72+1.34=74.99 .
\end{aligned}
$$

Note that the concept described above is not equivalent to the concept of "curve length" used by Katz [1988]. In order to compute "curve length", roughly representing the visual length of a graph [see also Sohrab, 2003, p. 295,

Problem 49], we would have to evaluate $\int_{a}^{b}\left\|\frac{d}{d x}(x, f(x))\right\|_{2} d x$, where the first component represents the dimension of time with a linear progression (see also caveats in [Raghavendra and Dutt, 2009, Castiglioni, 2010]). For a twodimensional graph, this equals $\int_{a}^{b}\left(1+\left(f^{\prime}(x)\right)^{2}\right)^{1 / 2} d x$, which is more complicated to evaluate, and in particular, where a constant function has a total variation of 0 on $[a, b]$, it will obviously have a curve length of $b-a$. 


\subsection{Total variation compared to other measures}

Total variation differs in several ways from traditional measures such as mean and variance. We illustrate this using the cumulative means and variances of several toy signals. For every point on the abscissæ of Figures 1b, 1c and 1d, the respective ordinate corresponds to the mean, variance, or total variation of the original signal in Figure 1a from the beginning up to that point. This makes some of the properties of these measures more evident, although it is not usually done in practice.

\subsubsection{Mean}

As total variation is only concerned with the differences between adjacent data points, it is completely insensitive to changes in mean affecting all data equally. This means that baseline "offsets" do not change total variation at all.

On the other hand, the mean is not suited very well for the analysis of undulatory processes, as can be seen in Figures $1 \mathrm{~b}$ and $1 \mathrm{~d}$, showing the cumulative means and total variations for the signals in Figure 1a. Although reflecting the undulatory structure of the original signals, the cumulative means quickly converge to zero, failing to convey but the most basic information about the signals analysed.

Another drawback of the mean of a set of data is its invariance under permutations of the input data, so that any information regarding the sequential nature of a process is lost: Any linear function on an interval as well as any permutation of it have the same mean, while their total variation is likely to be very different.

\subsubsection{Variance}

Any change in amplitude, frequency or additional undulations may have an influence on total variation, which is not necessarily the case for variance and in particular, total variation is not directly related to variance.

This can be seen comparing Figures $1 \mathrm{c}$ and 1d, where the cumulative variances and total variations for the signals in Figure 1a are shown. As it was already the case for the cumulative means, the culumative variances converge to a certain value, revealing little information about the structure of the original signals. On the other hand, Figure 2 shows that signals having different variance may have identical total variation, nonetheless.

Variance shares the behaviour of the mean under permutation of the input data, and thus cannot reveal features based on the sequence of data points.

\section{Applications to real data}

The following two subsections describe applications of total variation (TV) to two datasets which were already analysed with respect to different features. 

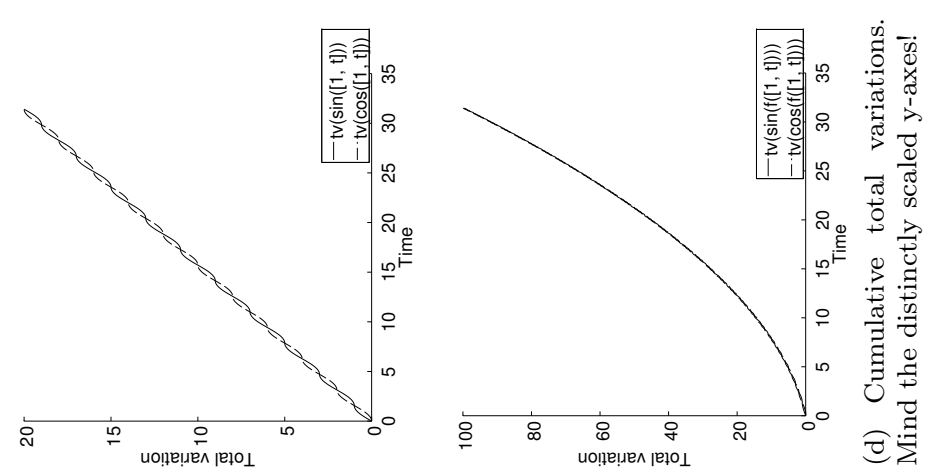

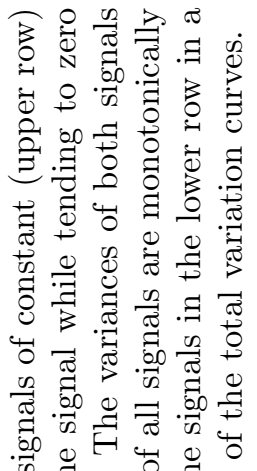
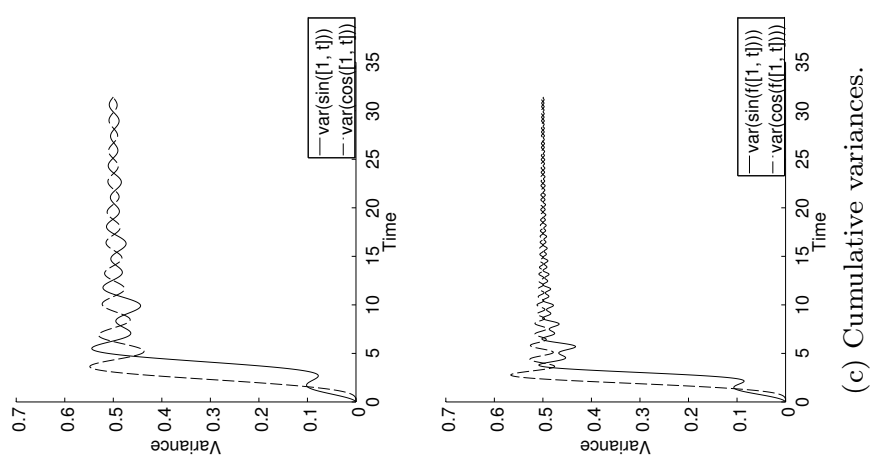

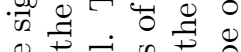

.

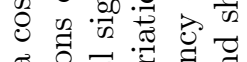

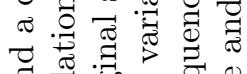

䨔

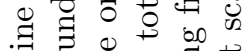

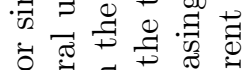

矛预.

.

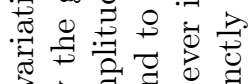
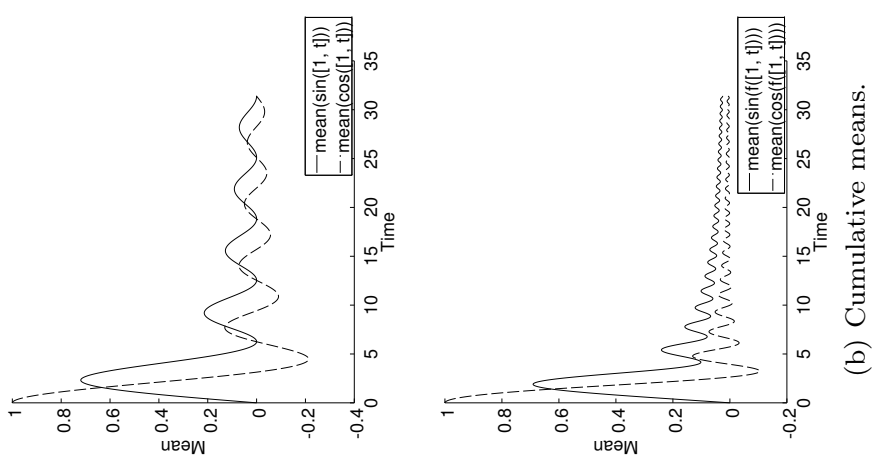

๙ 웅 चี ४ द्व

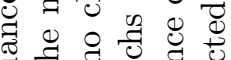
宁

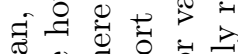
过 ¿乙

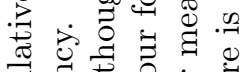
ప్ష
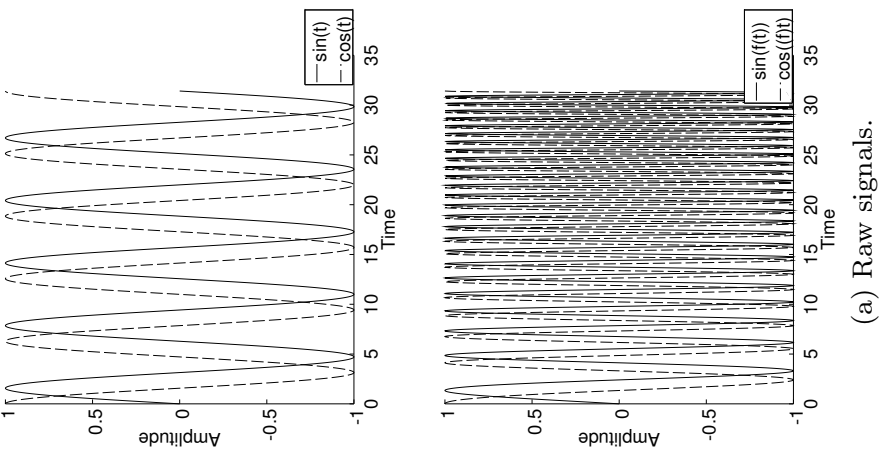

$0.50 \%$

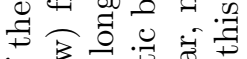

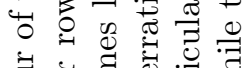

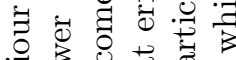
.

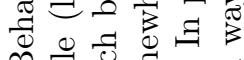

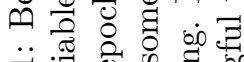
$\ddot{-1} \cdot \frac{\sigma}{\vec{*}}$ 요 $\stackrel{\circ}{>}=$

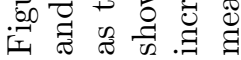



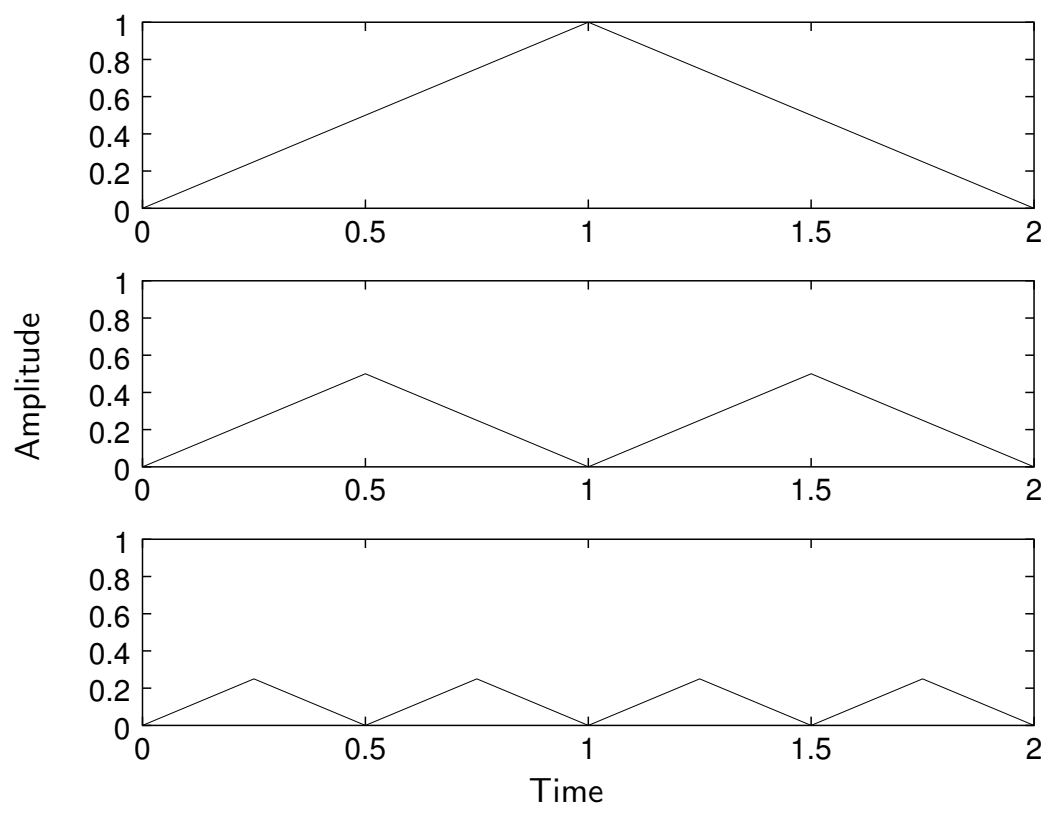

Figure 2: Three different functions with identical total variation and decreasing variance from top to bottom.

In both applications we will analyse the logarithm of TV instead of the raw measure, as the latter would tend to have skewed histograms, while the logarithm did not have this property. The assumption of homoscedasticity for the ANOVAs was backed up by the results of individual Bartlett-tests for each channel.

EEG-recordings were performed in both cases using a lab-specific electrode cap by EasyCap (Herrsching, Germany) with a regularly spaced array of 30 electrodes. The array started $5 \%$ anterior to $F_{z}$ and extended in steps of $15 \%$ of the nasion-inion distance in saggital and lateral directions (see Figure 3). The electrode located $5 \%$ posterior to $C_{z}$ served as the recording reference. The EEG was amplified with a Braintronics ISO 1032 amplifier and digitised at a rate of $500 \mathrm{~Hz}$ with the time-constant for the input set to $0.1 \mathrm{~s}$. The data were then filtered with a digital bandpass in the range of $0.3 \mathrm{~Hz}-30 \mathrm{~Hz}$, epochs with blinks were excluded from further analysis by extreme value statistics [Klein and Skrandies, 2013], and other artefacts were removed after mapping the data back to a normalised distribution [van Albada and Robinson, 2007] and comparing the mean and variance of the epochs to the respective $p=0.01$ levels. Finally, the data were rereferenced to the average reference [Offner, 1950].

The experiments were approved by the local ethics committee, and the experimental procedures followed the tenets of the Declaration of Helsinki. All participants gave their informed consent. 


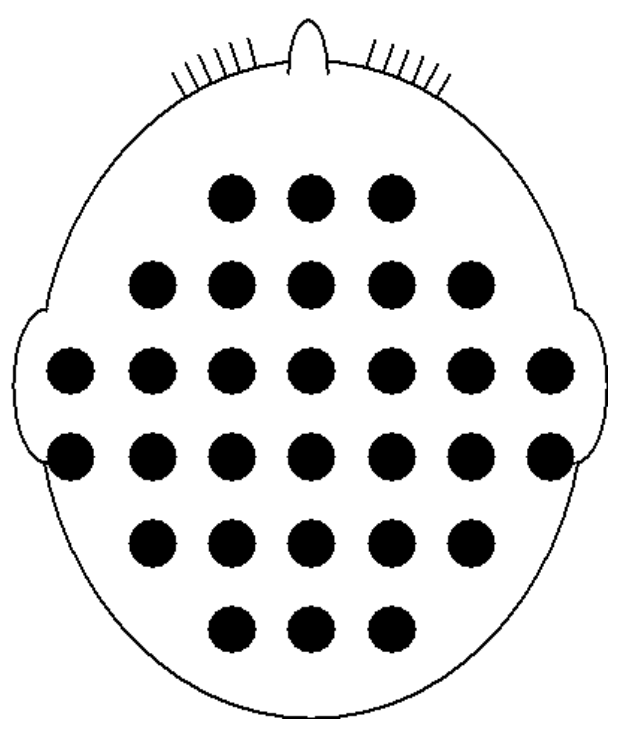

Figure 3: Electrode array as seen from above.

\subsection{Analysis of visually evoked potentials in a learning experiment}

\subsubsection{Experimental set-up and hypotheses}

We reanalysed data recorded for a doctoral dissertation [Würzer, 2013]. The original experiment was a study of participants learning the German meaning of a set of 40 Japanese Kanji-characters, which was counterbalanced by a set of 40 different Kanji not to be learned, thus serving as control stimuli. The stimuli are a superset of those used in Shinoda and Skrandies [2013], and are selected so as to cover different areas of semantics, and be comparable with respect to their psychovisual properties as well as their first appearance in the curriculum of Japanese primary schools.

Additionally, each participant was assigned to one of three groups, learning and reproducing the Kanji under the influence of different odours.

The experiment comprised two phases, where the participants were naïve to the meanings of all Kanji in the first phase, and had successfully completed the learning process by the second phase. The EEG of the participants was recorded during both phases and averaged in order to obtain the visually evoked potentials (VEPs) caused by presenting the Kanji on a computer screen.

\subsubsection{Data analysis}

Würzer [2013] analysed these data in the range of $0 \mathrm{~ms}-950 \mathrm{~ms}$ with respect to component latency, global field power (GFP) and centroid locations after defining suitable time windows based on the temporal variations of GFP. Within 
these windows, he found a number of main effects and interactions, but significant interactions of the factors "odour" and "experiment phase" were scarce. Hence, we collapsed the data across the factor "odour" in order to improve statistical power, and performed repeated measurement ANOVAs of the logarithmic $\mathrm{TV}$, using a hierarchical scheme of repeatedly halved time-windows.

\subsubsection{Results}

The results, shown in Figures 4-6, were surprising in that there was a highly significant main effect of learning- vs. control-stimuli, reaching significance levels lower than $10^{-6}$ (see Figure 4), while the main effects due to experiment phase were considerably less pronounced (Figure 5). The hierarchy of TV-analyses reveals that the interactions of both factors remained weak, while occurring quite late, towards the end of the stimulus interval of $1000 \mathrm{~ms}$ duration (Figure 6).

Looking at the VEPs in Figure 7, the only really obvious difference can be seen in Figure 7c, where quite obviously, TV should be higher for control- than for learning stimuli in the first time-window due to the considerable difference in peak-trough-amplitude. The features related to significant changes of TV in Figures $7 \mathrm{~b}$ and $7 \mathrm{c}$, however, are not prominent at all, and one would be tempted to readily dismiss the different patterns of undulation as changes of some residual noise, uncorrelated to the experimental manipulations. The ANOVA-results suggest a different interpretation, however:

Channel 13 bears a significant main effect of experiment phase in the last two segments, just failing to reach significance earlier. The most prominent features possibly affecting TV are subtle undulations in the signals, which become slightly more pronounced after learning.

Channel 27 bears a significant interaction between experiment phase and stimulus class. TV increases for control stimuli, while it decreases for learningstimuli, most likely due to changes in the undulatory structure of the signals. While it is certainly true that this interaction would have difficulty surviving a conservative correction of the respective $p$-values, it still remains interesting that the highest significance is reached when all four time-windows are considered at once. Hence, there might be some interaction of the factors hidden in a relatively slow process, but this would have to be confirmed independently.

Channel 30 bears a highly significant main effect of stimulus class in the first two segments. The features influencing TV most obviously are the P100 and the subsequent negative component, which are more pronounced for controlthan for learning-stimuli. In the second time-window, the overall undulations are stronger for control-stimuli as well. 


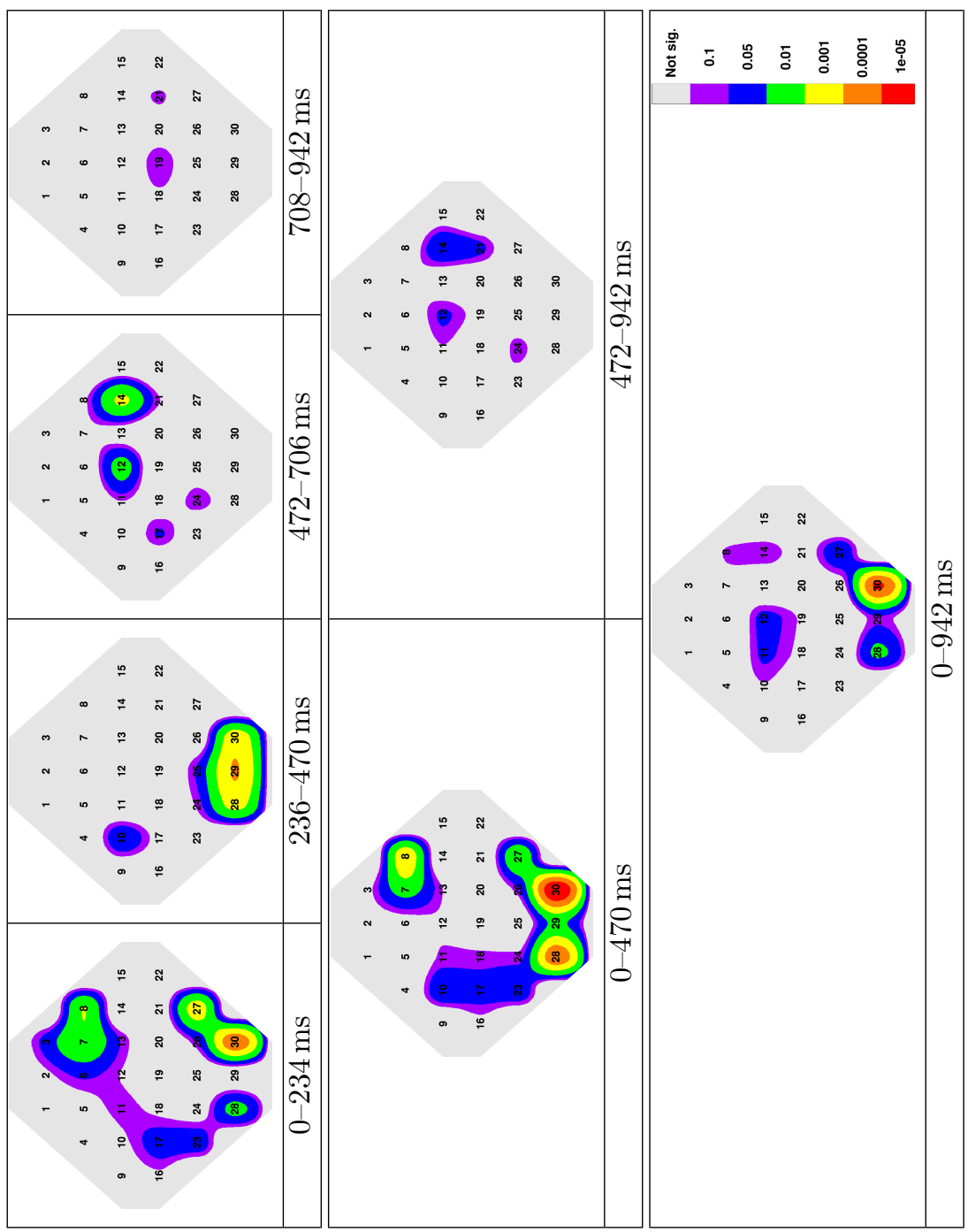

Figure 4: Main effects of stimulus class (learning vs. control). (Uncorrected $p$-values.) 


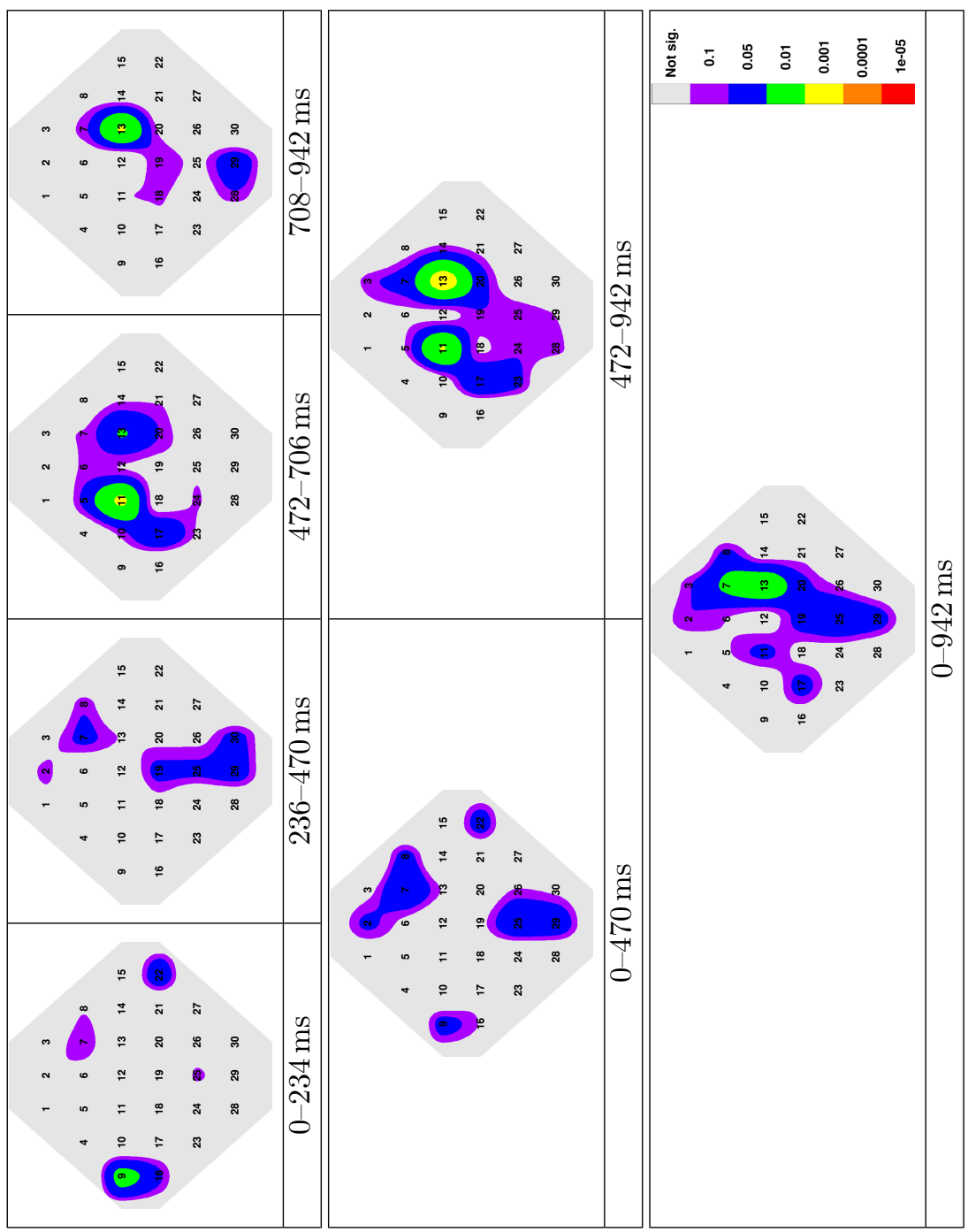

Figure 5: Main effects of experiment phase (before vs. after learning). (Uncorrected $p$-values.) 


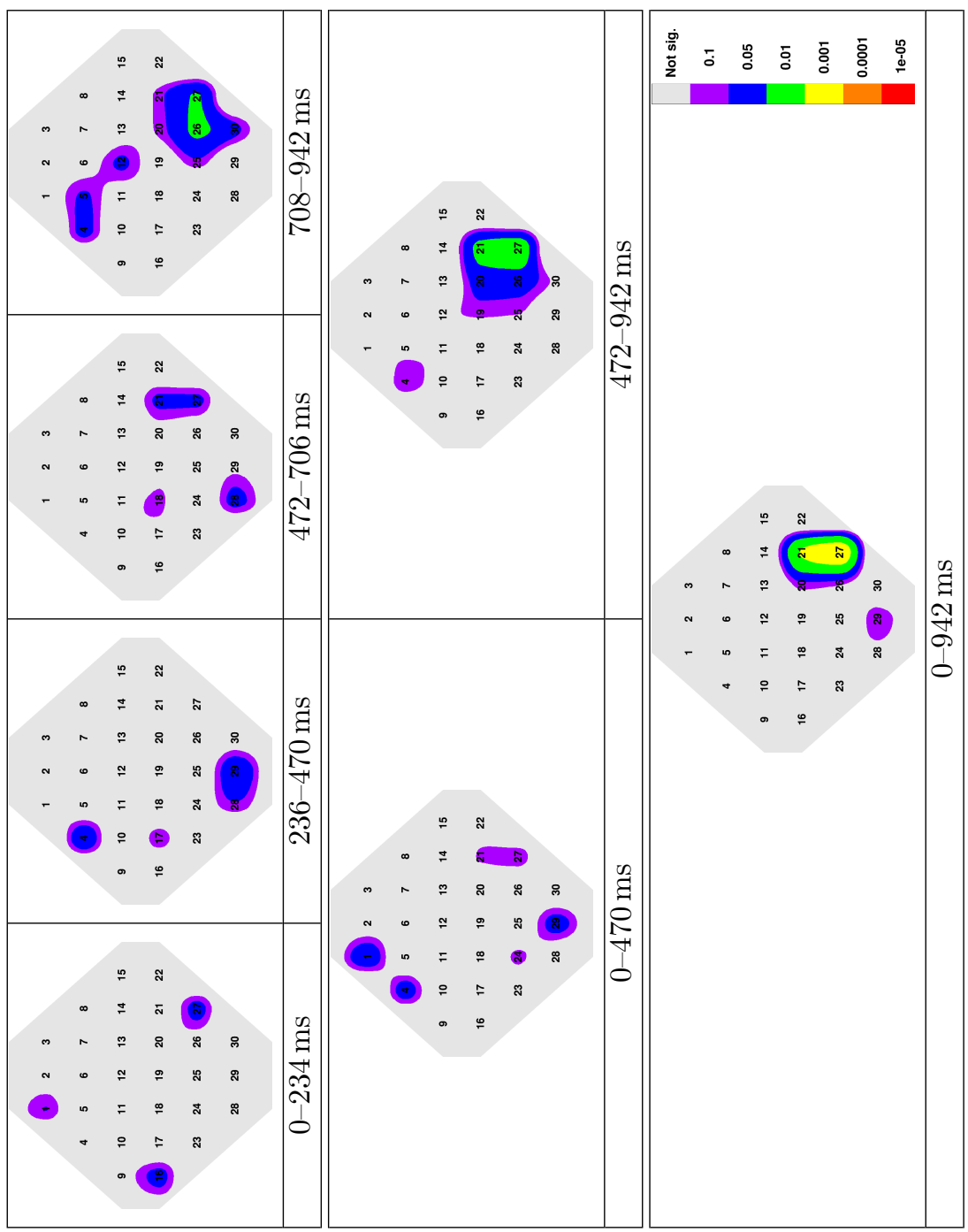

Figure 6: Interaction of stimulus class (learning vs. control) and experiment phase (before vs. after learning). (Uncorrected $p$-values.) 


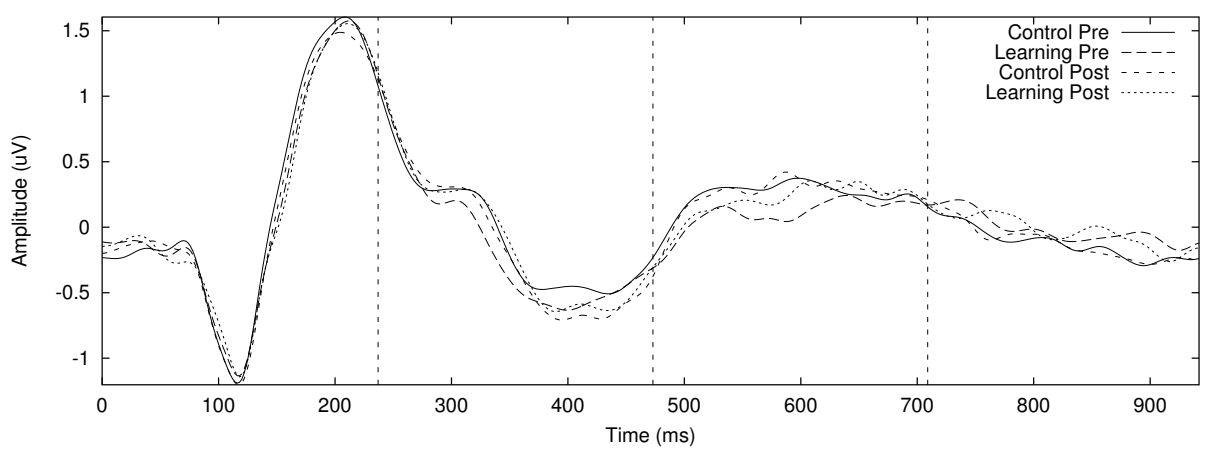

(a) Channel 13 (Most significant in Figure 5).

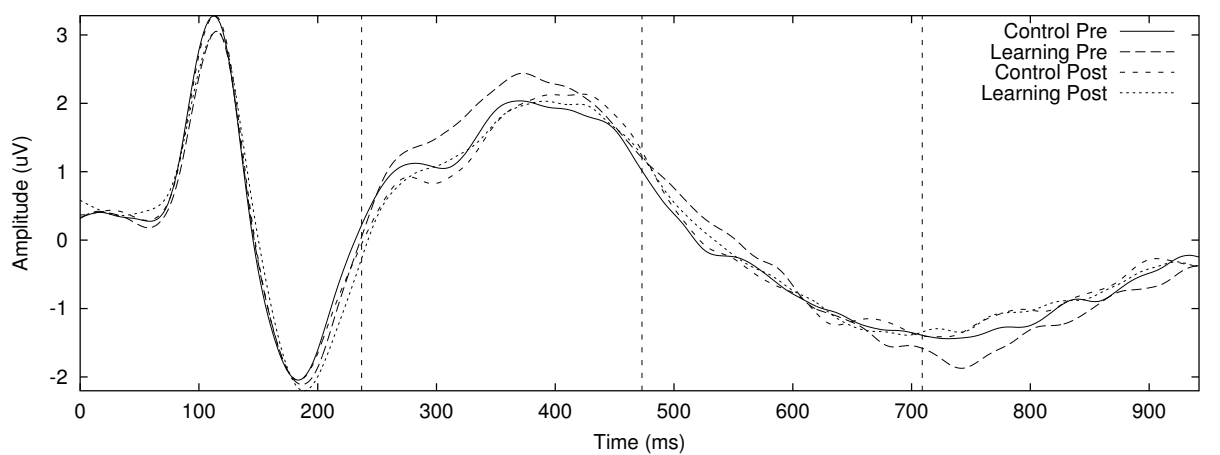

(b) Channel 27 (Most significant in Figure 6).

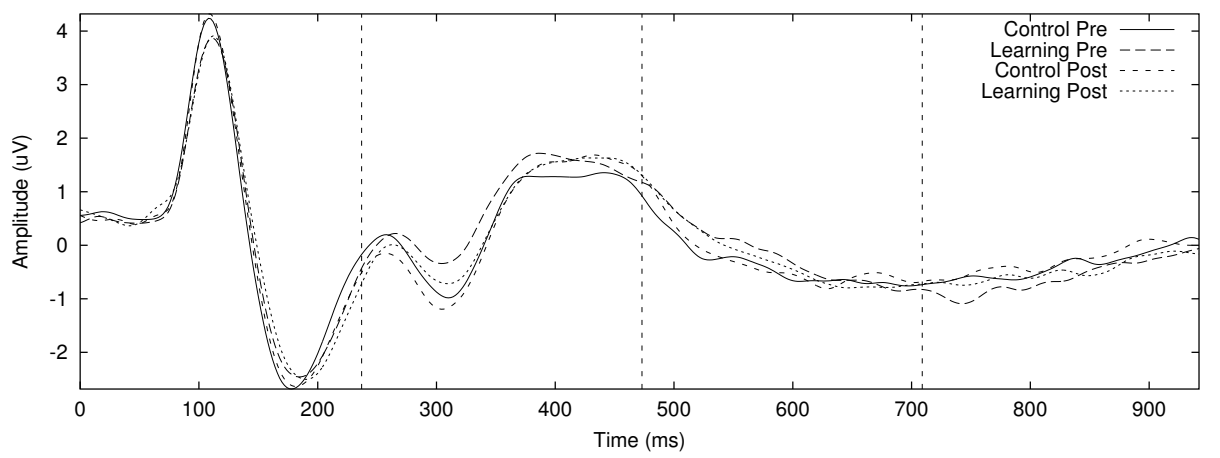

(c) Channel 30 (Most significant in Figure 4).

Figure 7: VEP waveforms for the most significant channels in Figures 4-6. As in those Figures, the time axis covers the range of $0 \mathrm{~ms}-942 \mathrm{~ms}$ post stimulus, and the dashed vertical lines mark the respective time-windows with borders at $235 \mathrm{~ms}, 471 \mathrm{~ms}$, and $707 \mathrm{~ms}$. 


\subsubsection{Discussion and conclusion}

TV-analysis reveals a number of main effects and interactions, many of them occurring not only in single, but rather in groups of adjacent channels. Main effects of stimulus class, for example, occur most significantly over the occipital cortex, but early and late effects occur over left parietal and right frontocentral areas as well. Significant main effects of experiment phase occur not quite as early over the occipito-parietal midline and later in the vicinity of the centroparietal midline, persisting until the end of the analysed interval, which is itself only some $50 \mathrm{~ms}$ shorter than the whole stimulus interval. Significant interactions of stimulus type and experiment phase occur surprisingly late over the right occipito-parietal cortex.

As we are more interested in exploratory data analysis, the general applicability of TV-analysis to EEG-data, and the usefulness of the method in general, we have not performed any conservative correction of the $p$-values. If a rigid method like Bonferroni-correction was to be applied to the individual maps, then roughly only those $p$-values inside the yellow areas would survive such a correction.

As the results stated by Würzer [2013, pp. 63-72] are concerned with measures analysing the topography of the whole matrix of channels, it is difficult to relate them directly to the results of $\mathrm{TV}$-analysis, which are available on the level of single channels, but after all, the TV-results seem plausible when looking at Würzer's Figure giving an overview of the most prominent components [Würzer, 2013, Fig. 13, p. 60].

To conclude, for the dataset at hand, TV-analysis provided us with

- the surprising result that - for whatever reason - there was an unexpected and highly significant difference in the participants' reactions to learningand control-stimuli, which was already hinted at by Würzer [2013], whose analyses could of course not reveal, which channels were involved,

- an easy way to "zoom" into the signal by means of hierarchical subdivision of the time-windows employed,

- some insight into the subtle differences of the signal's undulatory structures. It must be understood, however, that these changes need not be identical to those shown in Figure 7, as the grand mean can only show effects phase-locked across all participants; a limitation that TV-analysis is not subject to.

\subsection{Analysis of feedback-related potentials in a learning experiment}

\subsubsection{Experimental setup and hypotheses}

In another learning experiment [Skrandies and Klein, 2015], participants were given $3 \mathrm{~s}$ to rate the divisibility by $2,3,5,9$, or 11 , of randomly selected numbers 
comprising at most four digits, which were presented one at a time on a computer screen.

While divisibility of an integer by 2 and 5 is obvious from its last digit, divisibility by 3 is a bit more complicated, but can easily be assessed by summing up all digits and checking if the sum - which will usually have considerably fewer digits - is divisible by 3 , and most adults remember having learned this rule in school. There are similar rules for divisibility by 9 and 11, but they are not usually taught at German schools, and adults usually claim to not know them.

The experiment consisted of two phases: In the first phase, the participants were asked to solve a set of the above problems without having been instructed in the application of the according rules. Afterwards, rules for all of the above types of divisibility were explained to them, and they were asked to solve another set of tasks. During both phases, auditory feedback regarding the correctness of their answers was given to the participants, and the participants' EEG was recorded with markers indicating the beginning of task presentation and feedback presentation, respectively.

We hypothesised that learning the divisibility rules should impact the topography of the EEG upon task presentation in the second phase, and could show significant interactions of experiment phase and task difficulty by means of a wavelet transform [Skrandies and Klein, 2015].

As far as the auditory feedback is concerned, it is known that feeback-stimuli in general elicit certain event-related potentials. Negative feedback, in particular, elicits a feedback-related negativity $(\mathrm{FRN})$ in the vicinity of the frontoparietal midline [Miltner et al., 1997, Sailer et al., 2010], and feedback-related P 300-activity (fP 300) has been observerved, too, over frontal to parietal areas [Bellebaum and Daum, 2008, Sailer et al., 2010]. While there is ample literature on both components, we will not discuss them any further at this point, as our major aim is the evaluation of TV-analysis, rather than to relate our results to this previous research.

Anyway, we further hypothesised an impact of the factors "experiment phase" and "correctness" on the reaction to auditory feedback for difficult tasks, and in particular, we expected an interaction of the factors.

\subsubsection{Data analysis}

It turned out that the analysis of ERPs should become considerably more difficult for the feedback than it had been for the analysis of the tasks presented. Quite obviously, some of the participants were so irritated when receiving negative feedback that they repeatedly shook their heads, gnashed their teeth, or rolled their eyes, thus producing artefacts so strong and often as to render many of the recorded epochs unusable. In consequence, we lost 8 of 27 datasets, and the power of our statistical analyses was reduced accordingly.

Figure 8a shows the grand means of feedback-induced ERP-waveforms, where the FRN can be seen as a negative deflection in the curves of the "incorrect"conditions. However, even after the FRN has subsided, the curves continue to diverge, and there appears to be some weak P 300-like activity, which we 
Table 1: Durations of FRN and P1 before and after learning, as determinded from the curves in Figure 8b.

\begin{tabular}{ccccc}
\hline Component & \multicolumn{2}{c}{ FRN } & \multicolumn{2}{c}{ P1 } \\
\hline Learning & Before & After & Before & After \\
\hline \hline Duration $(\mathrm{ms})$ & 88 & 72 & 142 & 144 \\
\hline
\end{tabular}

will prudently name $\mathrm{P} 1$, to be seen as a broad undulatory complex from about $250 \mathrm{~ms}-400 \mathrm{~ms}$ in the "incorrect"-conditions again.

The differences can be seen in more detail in Figure 8b: Before learning, the classical FRN can be seen peaking in the difference waves at $226 \mathrm{~ms}$, and P1 has a double peak with its maximum at $336 \mathrm{~ms}$ (second peak). After learning, the FRN peaks at $224 \mathrm{~ms}$, and $\mathrm{P} 1$ reaches its maximum at $298 \mathrm{~ms}$ (first peak).

While the FRN has a defined peak in both phases of the experiment, thus offering itself for a direct analysis of raw amplitudes, this is not the case for $\mathrm{P} 1$, which shows several undulations in the ERPs that persist in the difference waves (see Figure 8b), and make an analysis of overall curve morphology seem to be a method preferrable to a mere analysis of amplitudes.

We determined the peak-latency of the FRN and P 1, and performed a preliminary repeated measurements ANOVA with factors "experiment phase" and "correctness" for raw amplitudes at these points in time. This revealed significant - though arguably obvious - main effects, but as far as significant interactions were concerned, the results were found wanting.

In the next step we analysed the TV of the ERP signals in the time-windows defined by the zeroes of the difference waves surrounding the FRN and P 1 components. Those windows differ considerably in length, however, and the question arises whether to normalise the computed TV-values by the length of the respective window as given in Table 1 . This decision may seriously affect the analysis in a way which will be discussed below.

\subsubsection{Results}

The ANOVA-results for both, the FRN and P 1 with and without normalisation, each, are aggregated in Figure 9. We have not tested whether any of the differences in significance level conveys any meaning itself [Gelman and Stern, 2006].

FRN Within this time-window there are highly significant main effects of correctness in the vicinity of the frontocentral midline, with the most significant effects occurring over the right hemisphere, and there are additional significant effects visible at lateral centroparietal to occipital sites over both hemispheres.

The presence of significant main effects of experiment phase depends strongly on whether or not TV is normalised to the length of the respective time-window before analysis. With normalisation, there is a number of significant main effects 


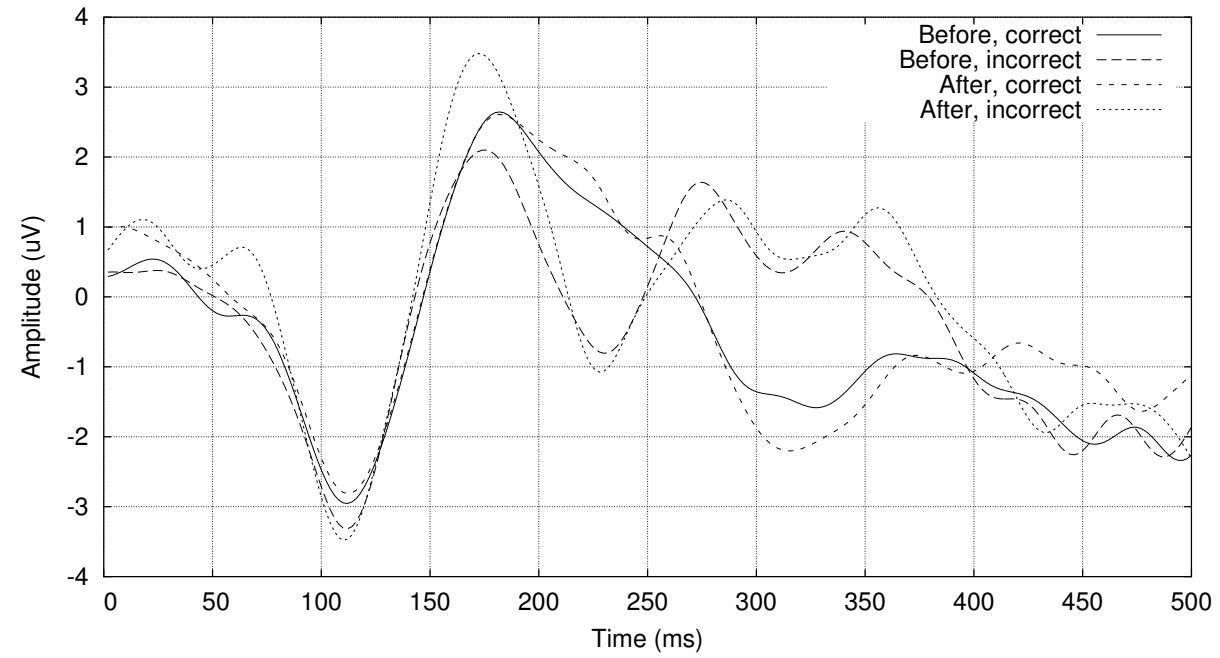

(a) ERPs to feedback "incorrect" show a pronounced negative deflection at about $220 \mathrm{~ms}$, and they also differ to ERPs induced by feedback "correct", although no obvious fP 300 is induced by any kind of feedback.

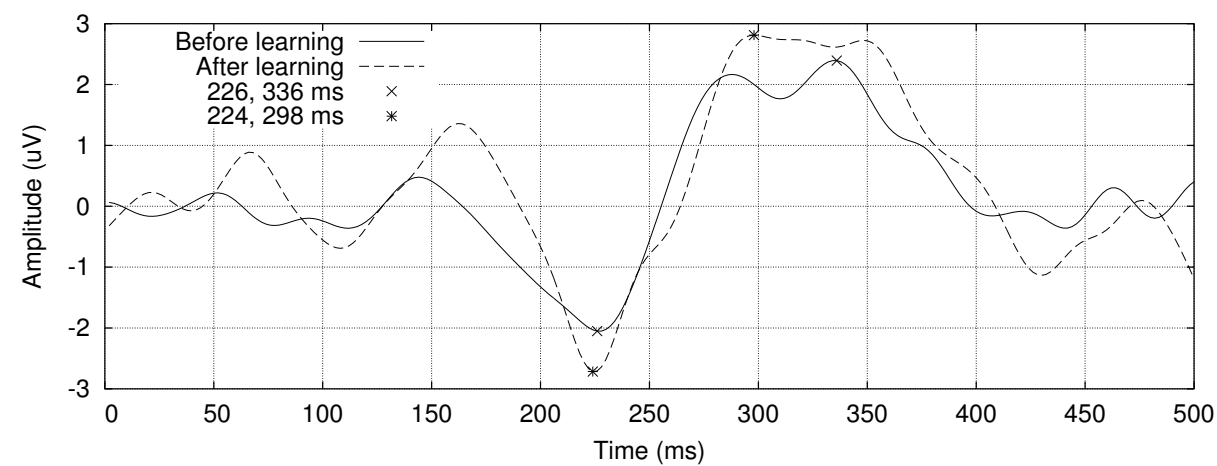

(b) Difference waves before and after learning: The time-course reveals activity for feedback "incorrect" in the form of a classical FRN and a following positive component (P 1), both relative to the feedback "correct" condition.

Figure 8: Grand means of raw ERPs and difference waves at electrode 6 (halfway between $F z$ and $C z$, see overview to the right). Feedback onset is at $0 \mathrm{~ms}$.

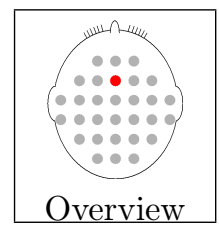


of experiment phase, which are generally located more anteriorly than those of correctness, but significant effects can be seen over occipital sites, too. All of these vanish, however, when normalisation is not used, while very few others appear.

Significant interactions of the factors "correctness" and "experiment phase" are present with, as well as without normalisation at lateral frontocentral to parietal sites as well as over the occipital midline.

P 1 Within this time-window there are significant main effects of correctness on a set of electrodes similar to the FRN-window, but as opposed to the latter, there seems to be a tendency towards the left hemisphere, and significance levels are more than an order of magnitude weaker. The main effects of experiment phase are less dependent on normalisation than in the FRN-window, and appear most significantly over frontocentral as well as over the mid to right occipital regions.

Significant interactions of correctness and experiment phase appear in centroparietal regions over both hemispheres.

\subsubsection{Discussion and conclusion}

As in the other experiment, TV-analysis revealed a number of interesting main effects and interactions. The latter results along with Figure 9, however, illustrate the substantial influence of normalisation on the ANOVA-results. As the lengths of the time-windows for FRN and P 1 differ, so do the normalisation factors for the data within, which results in different effects:

- ANOVA-comparisons within the same window are unaffected by normalisation, which is the case for main effects of correctness.

- Those between different windows may be substantially affected, which is the case for main effects of phase, but as a comparison between the results for the FRN and P1 shows, this need not be the case, and depends substantially on the difference in magnitude of the respective normalisation factors.

- The results for the interaction of factors are somewhat affected, but this cannot be seen in Figure 9, as the numbers are still very similar.

Quite obviously, different normalisation factors may lead to an unpredictable exaggeration or annihilation of effects, unless due care is taken, and consequently, comparing the TVs of time series of different original length cannot unequivocally be recommended. Such effects could be amended, perhaps, by introducing the normalisation factors as covariates and performing analyses of covariance instead, but this would have to be evaluated carefully and is beyond the scope of the current article. 


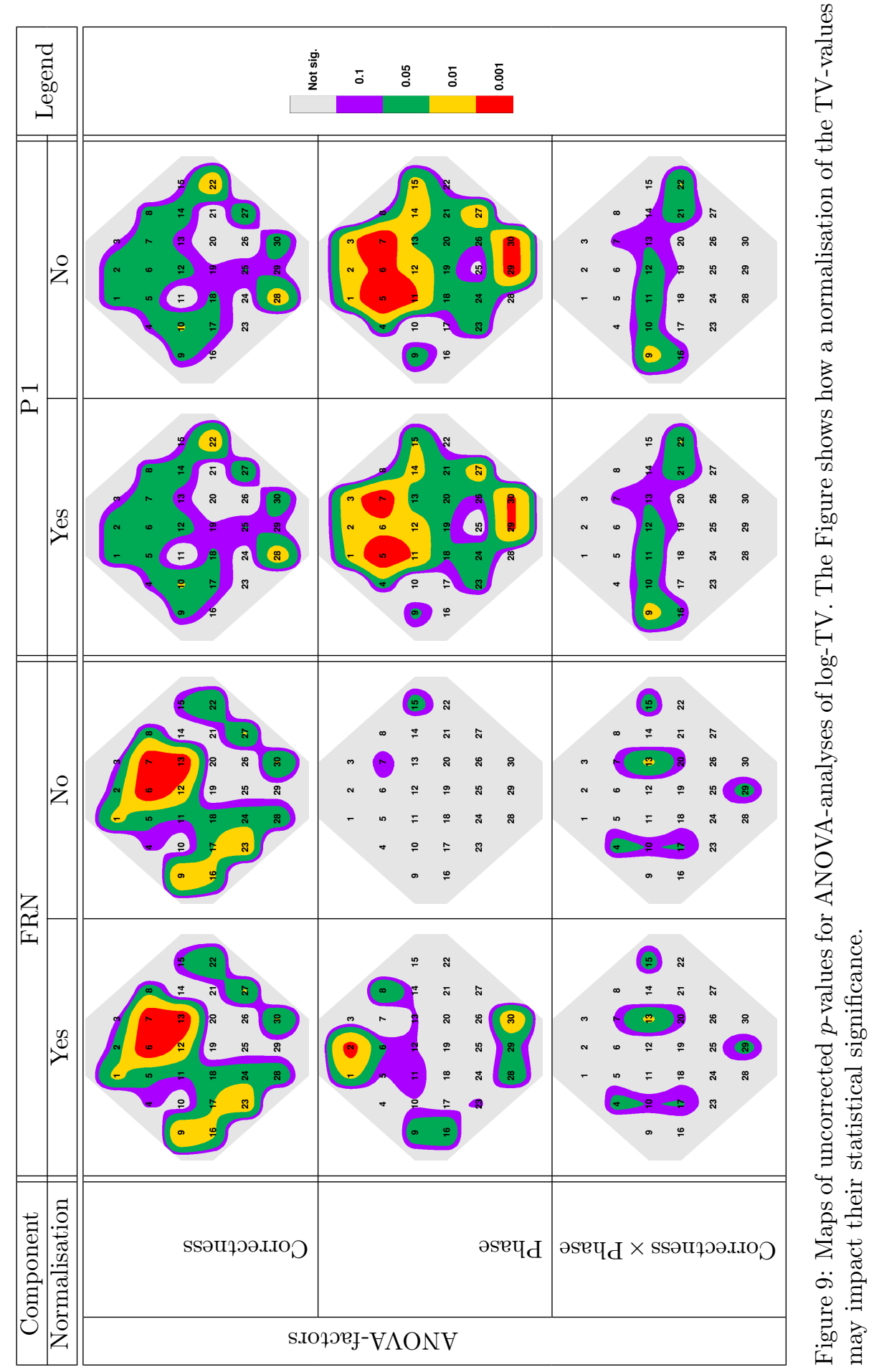




\section{Conclusion}

Our two applications of TV-analyses to real data have shown that TV may be used as a tool in situations, where experimental manipulations are expected to affect the morphology of a signal in ways not easily accessible by other standard measures. It can be used to conveniently condense a series of consecutive measurements into a single value accumulating information about several aspects of a signal (see Figure 1), which may otherwise go unnoticed. It can take into account morphological changes not necessarily phase-locked to an event, and as only differences of adjacent data are used in its computation, it is independent of baseline amplitude.

It should be clear, however, that it is yet one more tool, rather than being a panacea intended to replace a substantial amount of old tools. TV may be valuable on its own, but it can as well be used as a kind of loupe to aid in identifying interesting signals - or portions thereof - warranting further analysis.

Still, open questions remain to be assessed: It is not clear, for example, how exactly changes in variance impact the TV of a given signal. This problem comes into play when computing the TV of signals which are themselves averages of other signals, such as in a comparison of evoked activity across subjects. It may happen in this case that factors of an experiment have a systematic influence on the number of epochs to be averaged per subject, and as a consequence also on the residual noise to be found within the average, which may in turn lead to an increase of TV, not by way of changed curve-morphology, but rather by way of increased noise. It appears difficult to predict how such effects interact with changes introduced by experimental factors, and in order to avoid unwanted effects, additional covariates may have to be introduced.

\section{Conflicts of interest}

The authors declare that no conflicts of interest exist.

\section{References}

Christian Bellebaum and Irene Daum. Learning-related changes in reward expectancy are reflected in the feedback-related negativity. Eur. J. Neurosci., 27(7):1823-1835, Apr 2008. doi: 10.1111/j.1460-9568.2008.06138.x. URL http://dx.doi.org/10.1111/j.1460-9568.2008.06138.x.

Paolo Castiglioni. What is wrong in Katz's method? Comments on: "A note on fractal dimensions of biomedical waveforms". Comput. Biol. Med., 40 (11-12):950-952, 2010. doi: 10.1016/j.compbiomed.2010.10.001. URL http: //dx.doi.org/10.1016/j.compbiomed.2010.10.001.

Y. Deng, C. Wang, L. Chai, and Z. Zhang. Determination of Gabor wavelet shaping factor for accurate phase retrieval with wavelet- 
transform. Applied Physics B, 81(8):1107-1111, 2005a. ISSN 09462171. doi: 10.1007/s00340-005-1959-2. URL http://dx.doi.org/10.1007/ s00340-005-1959-2.

Yuqiang Deng, Zubin Wu, Lu Chai, Ching-yue Wang, Keisaku Yamane, Ryoji Morita, Mikio Yamashita, and Zhigang Zhang. Wavelet-transform analysis of spectral shearing interferometry for phase reconstruction of femtosecond optical pulses. Opt. Express, 13(6):2120-2126, Mar 2005b. doi: 10. 1364/OPEX.13.002120. URL http://www.opticsexpress.org/abstract. cfm?URI=oe-13-6-2120.

Matthias Dümpelmann, Julia Jacobs, Karolin Kerber, and Andreas SchulzeBonhage. Automatic 80-250 Hz "ripple" high frequency oscillation detection in invasive subdural grid and strip recordings in epilepsy by a radial basis function neural network. Clinical Neurophysiology, 123(9):1721 - 1731, 2012. ISSN 1388-2457. doi: http://dx.doi.org/10.1016/j.clinph.2012.02.072. URL http: //www.sciencedirect.com/science/article/pii/S1388245712001733.

Andrew Gelman and Hal Stern. The Difference between "Significant" and "Not Significant" Is Not Itself Statistically Significant. The American Statistician, 60(4):328-331, 2006. ISSN 00031305. URL http://www. jstor.org/stable/ 27643811 .

M. J. Katz. Fractals and the analysis of waveforms. Comput. Biol. Med., 18(3): 145-156, 1988.

Alexander Klein and Wolfgang Skrandies. A reliable statistical method to detect eyeblink-artefacts from electroencephalogram data only. Brain Topogr., 26(4): 558-568, Oct 2013. doi: 10.1007/s10548-013-0281-2. URL http://dx.doi. org/10.1007/s10548-013-0281-2.

D. Maraun, J. Kurths, and M. Holschneider. Nonstationary Gaussian processes in wavelet domain: Synthesis, estimation, and significance testing. Phys. Rev. E, 75:016707, Jan 2007. doi: 10.1103/PhysRevE.75.016707. URL http: //link.aps.org/doi/10.1103/PhysRevE.75.016707.

W. H. Miltner, C. H. Braun, and M. G. Coles. Event-Related Brain Potentials Following Incorrect Feedback in a Time-Estimation Task: Evidence for a "Generic" Neural System for Error Detection. J. Cogn. Neurosci., 9(6):788798, Nov 1997. doi: 10.1162/jocn.1997.9.6.788. URL http://dx.doi.org/ 10.1162/jocn.1997.9.6.788.

Octave community. GNU/Octave, 2012. URL www.gnu.org/software/ octave/.

F. F. Offner. The EEG as potential mapping: the value of the average monopolar reference. Electroencephalogr Clin Neurophysiol, 2(2):213-214, May 1950. 
B. S. Raghavendra and D. Narayana Dutt. A note on fractal dimensions of biomedical waveforms. Comput. Biol. Med., 39(11):1006-1012, Nov 2009. doi: 10.1016/j.compbiomed.2009.08.001. URL http://dx.doi.org/10.1016/j. compbiomed.2009.08.001.

Uta Sailer, Florian Ph. S. Fischmeister, and Herbert Bauer. Effects of learning on feedback-related brain potentials in a decision-making task. Brain Res., 1342:85-93, Jun 2010. doi: 10.1016/j.brainres.2010.04.051. URL http://dx. doi.org/10.1016/j.brainres.2010.04.051.

Haruo Shinoda and Wolfgang Skrandies. Topographic changes in event-related potentials because of learning of meaningful Kanji characters. Neuroreport, 24(10):555-559, Jul 2013. doi: 10.1097/WNR.0b013e3283623743. URL http: //dx.doi.org/10.1097/WNR.0b013e3283623743.

Wolfgang Skrandies and Alexander Klein. Brain activity and learning of mathematical rules-effects on the frequencies of EEG. Brain Res., 1603:133-140, Apr 2015. doi: 10.1016/j.brainres.2014.11.015. URL http://dx.doi.org/ 10.1016/j.brainres.2014.11.015.

H.H. Sohrab. Basic Real Analysis. Birkhäuser Boston, 2003.

S. J. van Albada and P. A. Robinson. Transformation of arbitrary distributions to the normal distribution with application to EEG test-retest reliability. $J$. Neurosci. Methods, 161(2):205-211, Apr 2007. doi: 10.1016/j.jneumeth.2006. 11.004. URL http://dx.doi.org/10.1016/j.jneumeth.2006.11.004.

M. Wacker and H. Witte. Time-frequency techniques in biomedical signal analysis. a tutorial review of similarities and differences. Methods Inf Med, 52(4): 279-296, 2013. doi: 10.3414/ME12-01-0083. URL http://dx.doi.org/10. 3414/ME12-01-0083.

Herbert Witte, Peter Putsche, Claudia Hemmelmann, Christoph Schelenz, and Lutz Leistritz. Analysis and modeling of time-variant amplitude-frequency couplings of and between oscillations of EEG bursts. Biol Cybern, 99(2):139157, Aug 2008. doi: 10.1007/s00422-008-0245-x. URL http://dx.doi.org/ $10.1007 / \mathrm{s} 00422-008-0245-\mathrm{x}$.

Benjamin Würzer. Visuell evozierte Potentiale und ihre Topographie beim Lernen japanischer Symbole unter Geruchseinflüssen. MD thesis, JustusLiebig-Universität, Otto-Behaghel-Str. 8, 35394 Gießen, 2013. URL http: //geb.uni-giessen.de/geb/volltexte/2014/11170. 